\title{
Image enhancement algorithm combining multi-scale Retinex and bilateral filter
}

\author{
WANG Ming-rong ${ }^{1}$, Jiang Shu-qing ${ }^{2}$ \\ College of Computer Science, Leshan Normal University, Leshan, China, 614004 \\ A Project Supported by Scientific Research Fund of Sichuan Provincial Education department,
}

Number:13ZB0105

Name: Enhancement Algorithm of Image Based on Multi-scale Retinex

\begin{abstract}
Keywords: blurred image; image enhancement; color constancy theory; defog
\end{abstract}
\begin{abstract}
Under some special conditions, the quality of the inquired image is low, and the image has blurred characteristic, in order to improve the quality of the blurred image, this paper proposes a blurred image enhancement algorithm based on improved multi-scale Retinex. The shortcomings of the traditional methods are analyzed, by the idea of constraining weighted average to optimize traditional algorithm, and enhance detail information of blurred image, and multiple blurred image are utilized on the algorithm performance simulation test. The simulation results show that the blurred image enhancement algorithm of improved multi-scale Retinex, solves the problem of the traditional Retinex algorithm, speed up the running speed of the blurred image enhancement, makes the blurred image clearer, and obtain better visual effect.
\end{abstract}

\section{Introduction}

In the blurred conditions for image shooting, due to the impact of atmospheric scattering effects, the color and contrast of captured image degenerate, image quality is lower, adversely affect is produced to the outdoor video surveillance system, so the blurred image have to be processed, so as to improve the quality of the image, which has very important significance [1].

The main purpose of blurred image enhancement is to improve the image contrast and saturation, and keep chroma unchanged. The blurred image enhancement algorithm is divided into physical methods and image processing method currently [2]. The physical method requires the prior knowledge of sunny image at same scene, and some physical device, which is not convenient in practical applications [3]. Therefore, independence on the physical device, has become the main research direction of blurred image enhancement, the common methods include: histogram equalization, wavelet transform, homomorphic filtering and Retinex algorithm [4-7]. The histogram equalization method by increasing the detail contrast of the image, to improve the quality of the image, but the original image color is distorted; homomorphic filtering method can have better processing for the image of not uniform illumination, but has poor effect on blurred image [8]. The Retinex theory of color constancy proposed by Edwin Land, can enhance the contrast of the image, and provide a new enhancement method to solve the blurred image quality problems [9]. However, a large number of studies show that, the image output by Retinex theory have some defects, like halo and over enhancement, the complexity of the algorithm is high, and can lead to RGB color offset to gray [10]. Then, some scholars have put forward a series of Retinex image improved enhancement algorithm, to some extent improve the blurred image quality, the enhancement effect is superior to the traditional Retinex algorithm, but the algorithm complexity is much higher [11]. Zhao Quanyou etc. obtained better multi-scale enhancement effect with the nonlinear Sigmoid, but it can only enhance dark region, and also not suitable for blurred image enhancement [12].

\section{The traditional Retinex image enhancement algorithm and defects}

In the traditional method, dark channel enhancement method is utilized for a scene image shoot under the fog environment, but the enhancement effect is not good. Therefore, this paper proposes 
blurred video image enhancement algorithm of improved multi-scale weighted average Retinex algorithm. The multi-scale weighted average Retinex algorithm is to determine a preferred enhanced block from many blocks need to be enhanced with regarding the edge pixels as the center, the image texture structure information in unit time is $G(x, y ; t)$, intuitionistic blurred sets of image texture subspace are defined as conduction function:

$$
p(x, t)=\lim _{\Delta x \rightarrow 0}\left[\sigma \frac{u-(u+\Delta u)}{\Delta x}\right]=-\sigma \frac{\partial u(x, t)}{\partial x}
$$

Adopting multi-scale weighted average Retinex algorithm to process adaptive enhancement for gray pixels value of image, as:

$$
\begin{aligned}
x_{i}(t)= & \sum_{k=1}^{p} \sum_{l=0}^{2} \varphi_{k l}\left[w_{i 1}^{l}, \cdots, w_{i n}^{l}\right]\left[x_{1}(t-k), \cdots, x_{n}(t-k)\right]^{T} \\
& -\sum_{k=1}^{q} \sum_{l=0}^{2} \theta_{k l}\left[w_{i 1}^{l}, \cdots, w_{i n}^{l}\right]
\end{aligned}
$$

The multi-scale weighted average Retinex characteristics is extracted as a weighted vector to guide atomizing image realize defog treatment with box filter, the control output of image after denoising is obtained as:

$$
\left\{\begin{array}{c}
V_{i}^{d}(t+1)=W \cdot V_{i}^{d}(t)+C_{1} \cdot R_{1} \cdot\left(P_{\text {best }}^{d}(t)-P_{i}^{d}(t)\right) \\
+C_{2} \cdot R_{2} \cdot\left(G_{\text {best }}^{d}(t)-P_{i}^{d}(t)\right) \\
P_{i}^{d}(t+1)=P_{i}^{d}(t)+V_{i}^{d}(t+1)
\end{array}\right.
$$

Wherein: $V_{i}^{d}(t), V_{i}^{d}(t+1), P_{i}^{d}(t)$ and $P_{i}^{d}(t+1)$ respectively represent feature enhanced strength of the image of multi grid, the constrained optimization algorithm of multi-scale weighted average Retinex characteristics evolution is defined as:

$$
\begin{array}{cc}
\text { min imize } & f(\vec{x}) \quad \vec{x}=\left(x_{1}, x_{2}, \ldots, x_{n}\right) \in \mathfrak{R}^{n} \\
\text { subject to } & g_{j}(\vec{x}) \leq 0, j=1, \ldots, l \\
& h_{j}(\vec{x})=0, j=l+1, \ldots, p
\end{array}
$$

wherein: $x \in \Omega$;

$S$ - decision vector of constrained optimization evolutionary algorithm;

$\Omega$ - feasible region of constrained optimization evolutionary algorithm.

And through constrained evolutionary algorithms to optimize the results of defog continually, box filter designs as:

$$
\left\{\begin{array}{l}
G_{j}^{\max }(j \in\{1, \ldots, p\} \\
G_{j}^{\max }=\max _{i=1, \ldots, N}\left(G_{j}\left(\vec{x}_{i}\right)\right) \\
j \in\{1, \ldots, p\}
\end{array}\right.
$$

The enhancement is processed for standard characteristics of blurred video image, like brightness, contrast and structure similarity, based on the results of image defogging, constantly optimize the image, optimization coefficient is defined as:

$$
\left\{\begin{array}{l}
G_{j}^{\max }(j \in\{1, \ldots, p\} \\
G_{j}^{\max }=\max _{i=1, \ldots, N}\left(G_{j}\left(\vec{x}_{i}\right)\right) \\
j \in\{1, \ldots, p\}
\end{array}\right.
$$

Finally, enhancement results of brightness, contrast and image structure similarity of the image are as :

$$
\begin{aligned}
& a_{k}=\left(\sum_{k}+\varepsilon U\right)^{-1}\left(\frac{1}{|w|} \sum_{i \in w_{k}} I_{i} p_{i}-u_{k} \bar{p}_{k}\right) \\
& b_{k}=\bar{p}_{k}-a_{k}^{T} u_{k} \\
& q_{i}=\frac{1}{|w|}\left(\sum_{i \in w_{k}} a_{k} I_{i}+b_{k}\right)=\bar{a}_{i}^{T} I_{i}+\bar{b}_{i}
\end{aligned}
$$

Among them, $\sum_{k}$ is the variance of color matrix in window $w, \varepsilon$ is the correction parameter, $I_{i}$ is the pixel value of input image $I, p_{i}$ is the pixel value of filtered input image, through the 
processing, the algorithm is optimized, and the imaging quality and the ability to identify of blurred image are improved.

\section{Improved MSR algorithm}

Image weighted average transformation not only greatly compress the dynamic range of image pixel fluctuation, but also effectively extend the dark pixels in the compressed image, moreover, the logarithmic form is closer to the light perception of human visual system. The basic forms of expression is

$$
g(x, y)=c[I(x, y)]^{y}
$$

In the formula, $I(x, y)$ is the original image, the $g(x, y)$ is the image after power transformation, c and $\gamma$ are normal numbers.

The weighted average can select the optimal pixel in the image, so as to enhance the image contrast. When $\gamma=1$, the weighted average is transformed into linear transformation; when the $0<\gamma<1$, weighted average can extend the gray value in low pixel value zone of original image, and compress gray value of high pixel value zone; when $\gamma>1$, weighted average can enhance the bright area information in the original image.

The weighted average can enhance the dark region information of image, and effectively compress the dynamic range of the image, but can sometimes lead to over enhancement for the original image light region, high light area appears. In order to suppress the occurrence of high light area of image, the non-linear transformation is applied. That is,

$$
\begin{gathered}
I_{2}(x, y)=\left(\frac{2}{1+\exp (-m)}-1\right) \times 255-v(x, y) \\
m=k(v) \times \frac{v}{v+a \bar{v}} \\
k(v)= \begin{cases}7 & 0 \leq v \leq 60 \\
(v-600) / 70+7 & 60<v \leq 200 \\
(v-200) / 55+9 & 200<v \leq 255\end{cases}
\end{gathered}
$$

In the formula, $a$ is the adjustment coefficient, $v(x, y)$ is the gray value of the image, $\bar{v}$ represents the average gray value of the original image.

After the image dynamic range is compressed, sometimes the image is blurred, due to deficiencies of Gauss filtering algorithm, then, unsharp mask filtering algorithm can be adopted to eliminate the image blur, enhance image details. The Gauss ambiguity of the image can be expressed as:

$$
U(x, y)=I(x, y) * G(x, y)
$$

In the formula, $\mathrm{G}(\mathrm{x}, \mathrm{y})$ is a two-dimensional Gauss's function.

After the blurred image is obtained, unsharp mask filtering is expressed as:

$$
I_{E n}(x, y)=\alpha \times \frac{c}{2 c-1} I(x, y)-\beta \times \frac{1-c}{2 c-1} U(x, y)
$$

In the formula, the parameters $\alpha$ and $\beta$ are the adjustment parameters, then, unsharp mask filtering can be utilized to eliminate most of the low frequency information, while retaining most of high frequency information, and further enhance the image detail information.

The work process of blurred image enhancement algorithm based on improved scale Retinex is as follows:

(1) the weighted average is processed to the original image, so as to enhance details in the dark areas of the original image.

(2) using the unsharp mask filtering algorithm function and the original image to do convolution, the image after low pass filtering is obtained, namely the irradiation component $\mathrm{L}(\mathrm{x}, \mathrm{y})$.

(4) irradiation component of part of low frequency component is removed from the original image, the information after enhancement is obtained, namely $\mathrm{R}(\mathrm{x}, \mathrm{y})$;

(5) power and frequency transformation is processed for $\mathrm{R}(\mathrm{x}, \mathrm{y})$ to obtain the enhanced image I '(x, y). 


\section{Simulation experiments}

Simulation environment. In order to verify the identification performance of blurred image enhancement algorithm based on the improved scale Retinex, in the platform of Pentium $2.8 \mathrm{GHz}$ CPU, 3GB memory, the operating system Windows XP, using matlab2009 to program, and comparing the SSR algorithm and MSR algorithm. The performance measurement method of the combination of the subjective evaluation and objective evaluation is adopted, the blurred image need to be enhanced as shown in figure 3. Objective evaluation criteria utilizes measurement parameters based on statistical characteristics of the image proposed by Jobson et, L, C, H respectively represent changes in brightness, contrast and tone, defined as follows:

$$
\begin{aligned}
L & =\frac{\operatorname{mean}\left(g_{\text {out }}(x, y)\right)-\operatorname{mean}\left(g_{\text {in }}(x, y)\right)}{\operatorname{mean}\left(g_{\text {in }}(x, y)\right)} \\
C & =\frac{\operatorname{var}\left(g_{\text {out }}(x, y)\right)-\operatorname{var}\left(g_{\text {in }}(x, y)\right)}{\operatorname{var}\left(g_{\text {in }}(x, y)\right)} \\
H & =\frac{\operatorname{mean}\left(H_{\text {out }}(x, y)\right)-\operatorname{mean}\left(H_{\text {in }}(x, y)\right)}{\operatorname{mean}\left(H_{\text {in }}(x, y)\right)}
\end{aligned}
$$

In the formula, $g_{\text {in }}(x, y)$ is the image before processing, $g_{\text {out }}(x, y)$ is the image after processing, $H_{\text {in }}(x, y)$ and $H_{\text {out }}(x, y)$ respectively represents the hue component in HSV space of color image before and after the treatment, var() and mean() respectively represent the variance and the mean calculation.

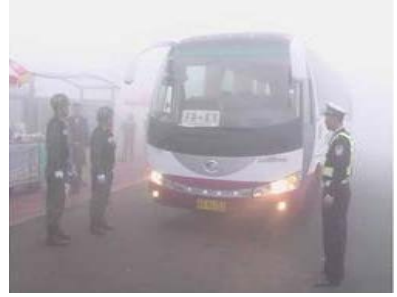

(a)

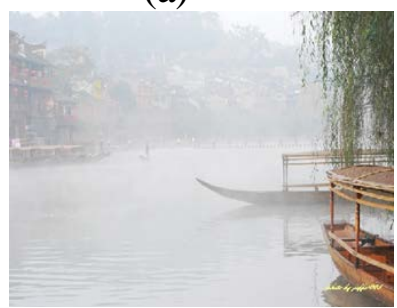

(c)

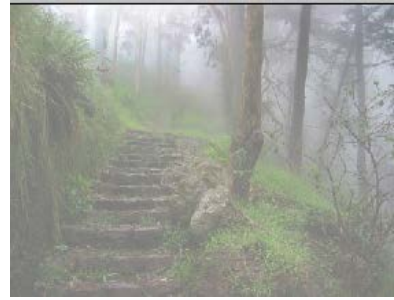

(b)

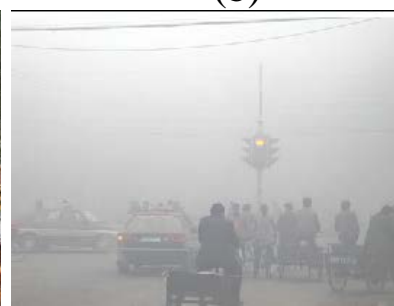

(d)

Figure 3 original image

Comparison of subjective performance. The face image enhancement effect with various algorithms are shown in figure 4 6. It can be known from Fig. 4 6 that:

(1) compared to the original blurred image, SSR algorithm can effectively remove the dark zone in image, but the excessive enhancement phenomenon exists.

(2) the MSR algorithm solves the defects of SSR algorithm caused by over enhancement, and enriches the details, there are still gray phenomenon and local dark situation.

(3) compared to other algorithms, blurred image enhancement algorithm based on improved scale Retinex can effectively eliminate image blur, the image is more clear, the image brightness and contrast are improved also, the image more consistent with human visual effect is obtained.

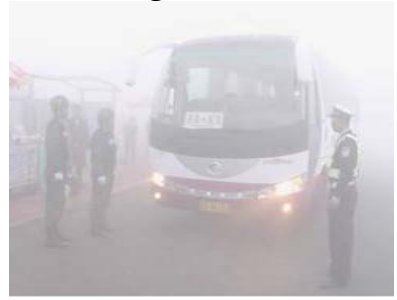

(a)

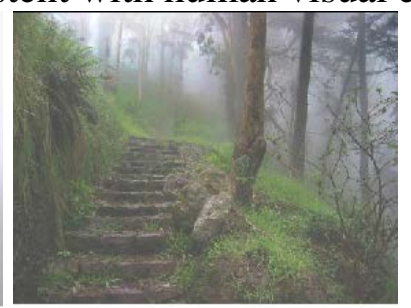

(b) 


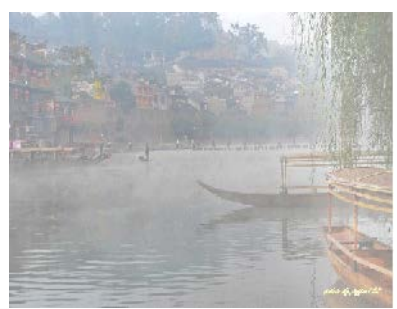

(c)

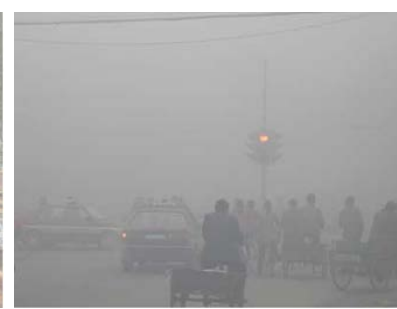

(d)

Fig. 4 the enhanced effect of the SSR algorithm

Comparison of the objective performance. Objective evaluation indexes of various algorithms are shown in Table 1, after analyzing table 1, it can be known that:

(1) the overall gray of SSR and MSR algorithm is relatively poor, the maintenance performance of tone is very poor, the contrast is also low.

(2) the dynamic range of blurred image enhancement algorithm based on improved scale Retinex is wide, image brightness is moderate, better tone fidelity can be obtained, comprehensive performance is better than the comparison algorithm.

Table 1 Comparison of objective performance of each algorithm

\begin{tabular}{llll}
\hline $\begin{array}{l}\text { Image enhancement } \\
\text { algorithm }\end{array}$ & $L$ & $C$ & $H$ \\
\hline SSR r scale & 0.459 & 0.043 & 0.33 \\
MSR & 0.733 & 0.412 & 3.32 \\
Improved & 1.2326 & 0.233 & 0.432 \\
Retinex algorithm & & & 0.33 \\
\hline
\end{tabular}

\section{Conclusions}

Aiming at the shortcomings of the traditional Retinex algorithm, a blurred image enhancement algorithm based on improved multi-scale Retinex is proposed. The power transformation is introduced to realize the dynamic range compression of the image, then a nonlinear transform is adopted to suppress the high light area of the image, and finally the unsharp mask filter is used to replace Gauss filter function to do convolution with the original image. Simulation results show that, the improved algorithm is a good solution to the problems of the traditional Retinex algorithm, image contrast is improved, and image details is enhanced, and obtain better reinforced effect, fast operation, very suitable for real-time image processing and analysis system.

\section{References}

[1] RUSSO F. An Image Enhancement Technique Combining Sharpening and Noise Reduction [J]. IEEE Transactions on Instrumentation and Measurement, 2002, 51(4): 824-828.

[2] Yu Jing, Xu Dongbin, Liao Qingmin. Research progress of the image haze removal technology [J]. Journal of image and graphics, 2011, 16 (9): 1561-1576.

[3] Zhou Xuan, Zhou Shudao. Research of haze removal technology for satellite images [J]. Computers application and software, 2005, 22 (12): 54-56.

[4] Chen Gong, Wang Tang, Zhou Heqin. A new method for image in foggy day based on the physics model [J]. Journal of image and graphics 2008, 13 (5): 888-893.

[5] Ron Kimmel, Michael Elad, Doron Shaked, Renato Keshet, and Irwin Sobel. A Variational framework for Retinex [J]. International Journal of Computer Vision, 2003, 52(1): 7-23.

[6] Rahman Z, Jobson D J, Woodell G A. Retinex Processing for automatic image enhancement [J]. Journal of Electronic Imaging, 2004, 13(1): 100-110.

[7] He K M, Sun J, Tang X O. Single image haze removal using dark channel prior[C]. Proceedings of IEEE Conference on Computer Vision and Pattern Recognition. Florida: IEEE, 2009: 19561963.

[8] Huang Lihong. A method degraded image contrast enhancement algorithm in foggy day based on single scale Retinex [J]. Journal of Applied Optics, 2010, 31 (5): 728-733. 
[9] Zhao Xiaoxia, Wang Rulin, Li Xueyan. Degraded image enhancement algorithm in foggy day based on Multi scale Retinex [J]. Industrial automation, 2009, 10:62-66

[10] Chen J, Paris S, Durand F. Real-time edge-aware image processing with the bilateral grid [J]. ACM Transactions on Graphics, 2007, 26(3): 103-111.

[11] Rui Yibin, Li Peng, Sun Jintao, et al. An interactive image defogging method [J]. Computer application, 2006, 26 (11): 2733-2735.

[12] Li Guanzhang, Luo Wusheng, Li Pei. Image contrast enhancement to avoid the color distortion [J]. Computer simulation, 2008, 25 (10): 228-231. 